\title{
Superior Mesenteric and Inferior Mesenteric Artery Diameters in Colorectal Cancer
}

\author{
Feng $\mathrm{Chi}^{1}$, Xiang Wang ${ }^{2}$, Yuefu Lan $^{1}$, Liangxian Jiang ${ }^{1}$ and Shenkang Zhou ${ }^{1}$ \\ ${ }^{1}$ Department of Gastrointestinal Surgery, Taizhou Hospital, Zhejiang University, China \\ ${ }^{2}$ Department of Vascular Surgery, Taizhou Hospital, Zhejiang University, China
}

\begin{abstract}
Objective: To compare the superior mesenteric artery (SMA) and inferior mesenteric artery (IMA) diameters in colorectal cancer compared to control.

Study Design: Observational study.

Place and Duration of Study: Taizhou Hospital, Zhejiang University, Taizhou, China, from March 2019 to June 2020.

Methodology: A total of 203 diagnosed colorectal cancer patients and 40 patients as control subjects were reviewed. Patients were divided into three groups based on tumor location as the right colon, left colon, and rectal groups. The diameters were measured on axial computed tomography images independently by two observers.

Results: The SMA diameter did not differ between the right colon and control groups $(p=0.626)$. The IMA diameter was significantly higher in the left colon group than in the control group $(p=0.002)$, but there was no significant difference in the IMA diameter between the different tumour stages $(p=0.263)$. The IMA diameter was significantly higher in the rectal group than in the control group $(p<0.001)$. There was a significant increase in the IMA diameter from stage I to stage II rectal cancers $(p=0.022)$ and from stage II to stage III rectal cancers $(p=0.003)$. The IMA diameter did not differ between stage III and IV rectal cancers $(p=0.600)$. In locoregional rectal cancer patients, there was a significant correlation between the IMA diameter and tumour-node-metastasis stage $(p<0.001, r s=0.494)$.

Conclusion: Patients with rectal cancer and left colon cancer have a wider IMA diameter than patients without colorectal cancer. IMA diameter can be a potential marker for locoregional staging of rectal cancer.
\end{abstract}

Key Words: Superior mesenteric artery, Inferior mesenteric artery, Colorectal cancer, Markers, Diameter.

How to cite this article: Chi F, Wang X, Lan Y, Jiang L, Zhou S. Superior Mesenteric and Inferior Mesenteric Artery Diameters in Colorectal Cancer. J Coll Physicians Surg Pak 2020; 30(11):1161-1165.

\section{INTRODUCTION}

Colorectal cancer is one of the most common alimentary canal malignancies worldwide. ${ }^{1,2}$ In China, colorectal cancer is the third most common cancer, with rectal cancer specifically accounting for nearly one-third of the total incidence. ${ }^{3}$ The superior mesenteric artery (SMA) and inferior mesenteric artery (IMA) are two of the three unpaired branches of the abdominal aorta. ${ }^{4,5}$ Tumours in the right colon derive blood supply from the SMA, ${ }^{6}$ while those in the left colon and rectum derive blood supply from the IMA. ${ }^{6,7}$

To meet their increasing vascularisation needs, tumours release angiogenic chemical signals to create new blood vessels, by a phenomenon known as tumour angiogenesis. ${ }^{8}$

Correspondence to: Dr. Shenkang Zhou, Department of Gastrointestinal Surgery, Taizhou Hospital, Zhejiang University, 150 Ximen Street, Taizhou, Zhejiang 317000, P. R. China

E-mail: zhoushenkang@yeah.net

Received: September 21, 2020; Revised: October 15, 2020;

Accepted: October 21, 2020

DOI: https://doi.org/10.29271/jcpsp.2020.11.1161
Thus, we speculated that the artery feeding a tumour may dilate to accommodate the increase in blood flow. Previous studies found that the diameter of the superior mesenteric vein was increased in right colon cancer, and the diameter of the inferior mesenteric vein was increased in rectal cancer. ${ }^{9,10}$ However, no assessment of the SMA and IMA diameters in colorectal cancer has been reported.

Therefore, the aim of this study was to assess whether the SMA and IMA were dilated in colorectal cancer, and whether they could be of use as new staging criteria for colorectal cancer.

\section{METHODOLOGY}

The current study recruited consecutive patients with primary colorectal cancer from Taizhou Hospital, Zhejiang University (Taizhou, China) from March 2019 to June 2020. The study was approved by the Ethics Committee of the Hospital (Number: K20200807). All patients underwent contrast-enhanced computed tomography (CT) of the abdomen and pelvis by endoscopic biopsy after confirmation of the presence of colon or rectal cancer. Patients with synchronous cancers, those who had undergone neoadjuvant treatment, those who had a history of abdominal surgery, and those with severe atheros- 
clerosis of the abdominal aorta and its branches were excluded.

The inducted patients were divided into three groups based on the tumour location as the right colon, left colon, and rectal groups. Patients with tumours in the caecum, ascending colon, hepatic flexure, and right half of the transverse colon were included in the right colon group; patients who had tumours in the left half of the transverse colon, splenic flexure, descending colon, and sigmoid colon were included in the left colon group. Patients with rectal cancer were included in the rectal group. The control group included 40 patients with abdominal pain who had normal contrast-enhanced abdominopelvic CT findings. The clinicopathological characteristics of the patients such as age, sex, body mass index (BMI), and tumour-node-metastasis (TNM) stage ( $7^{\text {th }}$ American Joint Committee on Cancer classification) were recorded. ${ }^{11}$

A 64-slice CT scanner (Aquilion, Canon, Japan) was used on all patients, following a standardised protocol. They were injected intravenously with $90 \mathrm{ml}$ of iopromide $(370 \mathrm{mg} / \mathrm{ml}$, lopamiron 370; Bayer, Osaka, Japan) at a speed of $3.0 \mathrm{ml} / \mathrm{s}$. Abdominal and pelvic scans were obtained using the following parameters: 120 kVP, $220 \mathrm{mAs}$, and $0.5 \mathrm{~mm}$ slice thickness. The multiphase CT scanning protocol included unenhanced, arterial (30 s delay), portal venous ( 60 s delay), and equilibrium phases ( 120 s delay).

The arterial phase axial CT images were anonymised and assessed for the SMA and IMA diameters by two observers. The SMA diameter was measured approximately $5 \mathrm{~mm}$ distal to its origin in the right colon and control groups (Figure 1). The IMA diameter was measured approximately $5 \mathrm{~mm}$ distal to its origin in the left colon, rectal, and control groups (Figure 2). Each observer took three measurements using an electronic calliper on the imaging workstation. The resultant SMA and IMA diameters were reported as the average of six measurements to minimise measuring error.

Continuous variables were presented as mean \pm standard deviations and analysed using Student's t-test. Categorical variables were expressed as proportions and tested using chisquare test. The one-way analysis of variance and least significant difference test were used to compare continuous variables in multiple comparisons. The relationship among variables was assessed using the Spearman's rank correlation coefficient. The p-values $<0.05$ were considered to indicate statistical significance. Analyses were performed using IBM SPSS Statistics version 22.0 (IBM Co., Armonk, NY, USA).

\section{RESULTS}

A total of 203 patients were enrolled, of whom 23 patients with stage IV cancers were diagnosed by laparoscopic exploration or CT (visibility of characteristic findings of metastasis). The remaining 180 patients without metastasis underwent surgical resection and were staged postoperatively according to the pathology observed in the resected specimen.

There were 42 patients in the right colon group including 6
(14.3\%) patients with stage I cancer, 14 (33.3\%) with stage II cancer, 16 (38.1\%) with stage III cancer, and 6 (14.3\%) with stage IV cancer. Age, sex, weight, and BMI did not differ between the right colon group and the control group (all $p>0.05$ ). There was no significant difference in the SMA diameter between the right colongroup and the control group ( $p=0.626$, Table I).

Table I: Comparison of clinicopathologic characteristics between right colon cancer patients and controls.

\begin{tabular}{|l|c|c|c|}
\hline Parameter & $\begin{array}{c}\text { Right colon group } \\
(\mathbf{n = 4 2})\end{array}$ & $\begin{array}{c}\text { Control group } \\
(\mathbf{n}=\mathbf{4 0 )}\end{array}$ & $\mathbf{p}$-value \\
\hline Age (years) & $58.57 \pm 12.38$ & $61.25 \pm 9.02$ & 0.268 \\
\hline Sex & $23(54.8 \%)$ & $21(52.5 \%)$ & 0.837 \\
Male & $19(45.2 \%)$ & $19(47.5 \%)$ & \\
Female & $60.26 \pm 11.58$ & $60.82 \pm 10.14$ & 0.816 \\
\hline Weight $(\mathrm{kg})$ & $22.60 \pm 3.55$ & $22.79 \pm 3.51$ & 0.801 \\
\hline BMI $\left(\mathrm{kg} / \mathrm{m}^{2}\right)$ & $5.32 \pm 0.90$ & $5.22 \pm 1.04$ & 0.626 \\
\hline SMA diameter & (mm) & \multicolumn{4}{|l|}{ BMI: Body mass index, SMA: Superior mesenteric artery. } \\
\hline
\end{tabular}

Table II: Comparison of clinicopathologic characteristics between left colon cancer patients and controls.

\begin{tabular}{|l|l|l|l|}
\hline Parameter & $\begin{array}{l}\text { Left colon group } \\
(\mathbf{n = 5 2 )}\end{array}$ & $\begin{array}{l}\text { Control group } \\
(\mathbf{n = 4 0 )}\end{array}$ & p-value \\
\hline Age (years) & $64.15 \pm 10.59$ & $61.25 \pm 9.02$ & 0.168 \\
\hline $\begin{array}{l}\text { Sex } \\
\text { Male }\end{array}$ & $25(48.1 \%)$ & $21(52.5 \%)$ & 0.674 \\
Female & $27(51.9 \%)$ & $19(47.5 \%)$ & \\
\hline Weight $(\mathrm{kg})$ & $59.49 \pm 10.41$ & $60.82 \pm 10.14$ & 0.539 \\
\hline BMI (kg/m $\left.{ }^{2}\right)$ & $22.51 \pm 3.25$ & $22.79 \pm 3.51$ & 0.686 \\
\hline $\begin{array}{l}\text { IMA diameter } \\
\text { (mm) }\end{array}$ & $2.48 \pm 0.38$ & 0.002 \\
\hline \multicolumn{4}{|l|}{ BMI: Body mass index, IMA: Inferior mesenteric artery. } \\
\hline
\end{tabular}

Table III: Comparison of clinicopathologic characteristics between rectal cancer patients and controls.

\begin{tabular}{|l|l|l|l|}
\hline Parameter & $\begin{array}{l}\text { Rectal group } \\
(\mathbf{n = 1 0 9 )}\end{array}$ & $\begin{array}{l}\text { Control group } \\
(\mathbf{n}=\mathbf{4 0})\end{array}$ & p-value \\
\hline Age (years) & $63.54 \pm 10.18$ & $61.25 \pm 9.02$ & 0.212 \\
\hline $\begin{array}{l}\text { Sex } \\
\text { Male }\end{array}$ & $70(64.2 \%)$ & $21(52.5 \%)$ & 0.194 \\
Female & $39(35.8 \%)$ & $19(47.5 \%)$ & 0.869 \\
\hline Weight $(\mathrm{kg})$ & $61.12 \pm 9.70$ & $60.82 \pm 10.14$ & 0.795 \\
\hline BMI (kg/m $\left.{ }^{2}\right)$ & $22.95 \pm 3.12$ & $22.79 \pm 3.51$ & $<0.001$ \\
\hline $\begin{array}{l}\text { IMA diameter } \\
\text { (mm) }\end{array}$ & $2.57 \pm 0.49$ & $2.20 \pm 0.47$ & \\
\hline BMI: Body mass index, IMA: Inferior mesenteric artery. &
\end{tabular}

There were 52 patients in the left colon group, including 9 (17.3\%) patients with stage I cancer, 19 (36.5\%) with stage II cancer, 17 (32.7\%) with stage III cancer, and 7 (13.5\%) with stage IV cancer. Age, sex, weight, and BMI did not differ between the left colon group and the control group (all $p>0.05$ ). The mean IMA diameter was significantly wider in the left colon group than in the control group ( $p=0.002$, Table II). The IMA diameters in patients with stage I, stage II, stage III, and stage IV left colon cancer were $2.28 \pm 0.33 \mathrm{~mm}, 2.46 \pm 0.32 \mathrm{~mm}$, $2.58 \pm 0.42 \mathrm{~mm}$, and $2.51 \pm 0.42 \mathrm{~mm}$, respectively. There was no significant difference in the IMA diameter between patients with different stages of cancer $(p=0.263)$. 


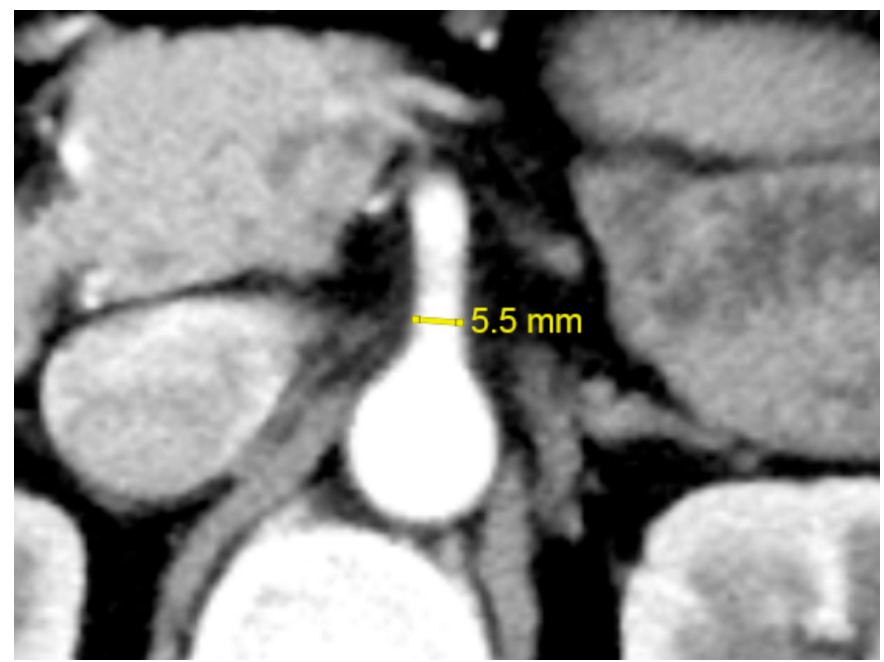

Figure 1: Measurement of the superior mesenteric artery (SMA) diameteron arterial-phase axial computed tomography (CT).

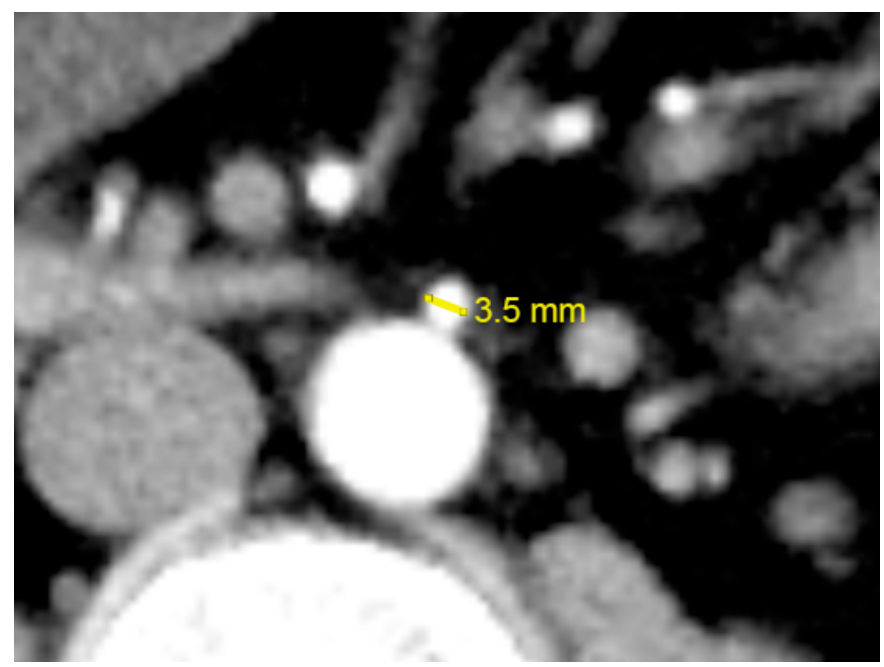

Figure 2: Measurement of the inferior mesenteric artery (IMA) diameter on arterial-phase axial computed tomography (CT).

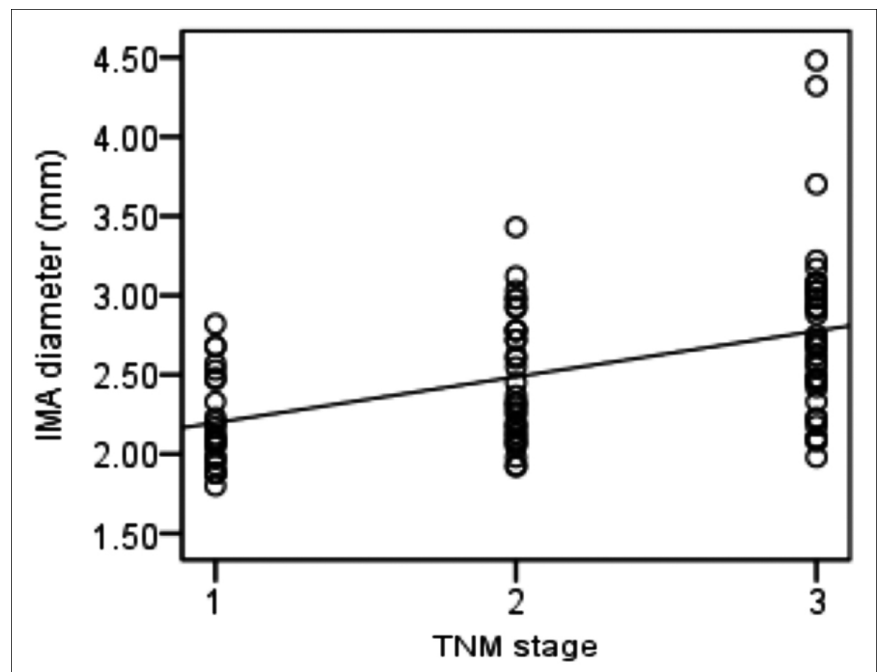

Figure 3: Correlation between TNM stage and inferior mesenteric artery (IMA) diameter in locoregional rectal cancer. Spearman's rank correlation analysis shows positive and moderate correlation between TNM stage and IMA diameter ( $r s=0.494, p<0.001)$. TNM stages 1,2 , and 3 (X-axis) represent stages I, II, and III, respectively.
There were 109 patients in the rectal group, including 24 $(22.0 \%)$ with stage I cancer, 34 (31.2\%) with stage II cancer, 41 (37.6\%) with stage III cancer, and 10 (9.2\%) with stage IV cancer. Age, sex, weight, and BMI did not differ between the rectal and control groups (all $p>0.05$ ). The mean IMA diameter was significantly wider in the rectal group than in the control group ( $p<0.001$, Table III). The IMA diameters in patients with stage I, stage II, stage III, and stage IV rectal cancer were $2.21 \pm 0.29 \mathrm{~mm}, 2.48 \pm 0.40 \mathrm{~mm}, 2.78 \pm 0.53 \mathrm{~mm}$, and $2.86 \pm 0.39 \mathrm{~mm}$, respectively $(p<0.001)$. There was a significant difference in the IMA diameter between patients with stage I and stage II cancers $(p=0.022)$, stage I and stage III cancers $(p<0.001)$, stage I and stage IV cancers $(p<0.001)$, stage II and stage III cancers $(p=0.003)$, and stage II and stage IV cancers $(p=0.015)$. However, there was no significant difference between patients with stage III and stage IV cancers $(p=0.600)$. In patients with locoregional (stages I-III) rectal cancers, there was a significant linear and moderate correlation between the IMA diameter and TNM stage ( $p<0.001, \mathrm{rs}=0.494$, Figure 3 ).

\section{DISCUSSION}

For colorectal cancer, imaging plays an important role in developing optimal treatment strategies by identifying the extent of local tumour growth and metastases. ${ }^{12,13}$ Patients with early tumours can proceed to surgery directly. However, patients with locally advanced tumours or metastasis usually require neoadjuvant chemoradiotherapy or palliative chemotherapy. ${ }^{14,15}$ Imaging modalities commonly used in the staging of colorectal cancer are CT, magnetic resonance imaging (MRI), and endoscopic ultrasonography (EUS). ${ }^{16,17}$ However, these modalities have limitations. CT exposes patients to radiation. MRI is relatively more expensive and time-consuming and is not applicable for patients with contraindications to MRI use. ${ }^{18}$ EUS is invasive, operator-dependent, and not applicable for patients with stenosis. ${ }^{19}$ Since the quality of preoperative staging could influence the treatment and prognosis of patients with colorectal cancer, there is an urgent need for new markers to increase the staging accuracy of this cancer.

In this study, it was hypothesised that the SMA and IMA might be dilated in colorectal cancer and thus, might be of use as new markers for colorectal cancer. This hypothesis was mainly based on the fact that tumour growth requires an increased blood supply than normal. In routine practice, SMA and IMA are easily visualised on contrast-enhanced CT, which is a routine test done on patients with colorectal cancer at the study centre. Hence, the authors used contrast-enhanced CT rather than angiography to measure the SMA and IMA diameters.

Since branches of the SMA supply the the right half of the colon, the SMA diameter between patients in the right colon cancer group and those in the control group were compared. The IMA supplies the left side of the colon and rectum; hence, the authors compared the IMA diameter between left colon cancer patients and rectal cancer patients and the control group.

It was found that there was no relationship between the pres- 
ence or absence of right colon cancer and the SMA diameter. Hence, the SMA diameter had no diagnostic significance in right colon cancer. However, it was found that the IMA diameter of patients with left colon cancer and rectal cancer was significantly higher than that of patients in the control group. This can be explained by the fact that the baseline diameter of the SMA is much wider than that of the IMA. Hence, the increased blood supply that the tumour induces has lesser influence on the SMA diameters than on the IMA diameter.

The IMA diameters were further compared in left colon cancer and rectal cancer patients with different TNM stages. There was a significant difference between the IMA diameter in rectal cancer patients with different TNM stages. However, no significant difference was found between the IMA diameter in left colon cancer patients with different TNM stages. The authors speculate that this may be due to the pattern of arterial supply to the left colon and rectosigmoid colon. The blood supply to the left colon and rectosigmoid region is from the branches of the IMA, namely the left colic artery, sigmoid arteries (could be two or three), and superior rectal artery (SRA). ${ }^{20}$ These arteries are connected by the marginal artery of Drummond, which further connects to the branches of the SMA. ${ }^{21,22}$ Left colon cancer is proximal to rectal cancer, which means that it is more likely for left colon cancer to derive part of its blood supply from the SMA through the marginal artery of Drummond than rectal cancer. In addition, there is a weak point in the colorectal blood supply known as Sudeck's point between the last sigmoidal branch and the SRA. ${ }^{23,24}$ This hinders the blood supply of IMA from other major arteries except the SRA. For these reasons, the IMA diameter could be a major factor for the growth of rectal cancer than left coloncancer.

There was a significant increase in the IMA diameter from stage I to stage II rectal cancers and from stage II to stage III rectal cancers. However, there was no significant difference between patients with stage III and stage IV cancers. This could be explained by the fact that stage I, stage II, and stage III rectal cancers are locoregional rectal cancers, all of which derive their blood supply from the IMA, but not in the case of metastatic tumours in stage IV rectal cancer. A moderate positive correlation was found between the IMA diameter and TNM stage in patients with locoregional rectal cancer. These findings suggest that the IMA diameter may be a new diagnostic marker for the locoregional staging of rectal cancer.

This study also had some limitations. First, this was a retrospective study conducted in a single centre, which might have led to selection bias. Second, the sample size was small. Third, the measurement error was also an important limitation. However, we minimised this by using two observers and considering the average of six measurements. Future, well-designed prospective studies are required to further support the findings of this study.

\section{CONCLUSION}

Patients with left colon cancer and rectal cancer had signifi- cantly an increased mean IMA diameter. In patients with locoregional rectal cancer, the IMA diameter increased with increasing TNM stage, suggesting the potential role of the IMA diameter in staging locoregional rectal cancer.

\section{FUNDING:}

The present study was supported by the Natural Science Foundation of Zhejiang Province (No. LGF19H160017).

\section{ETHICALAPPROVAL:}

The present study was approved by the Ethics Committee of Taizhou Hospital, Zhejiang University (Number: K20200807, Date: 15/08/2020).

\section{PATIENTS' CONSENT:}

A waiver for informed consent was obtained from the Ethics Committees of Taizhou Hospital, Zhejiang University; and all the data from patients were analysed anonymously.

\section{CONFLICT OF INTEREST:}

Authors declared no conflict of interest.

\section{AUTHORS' CONTRIBUTION:}

FC, XW: Conception and design, drafting of manuscript.

YL, LJ: Acquisition of data, Search of literature.

SZ: Analysis and interpretation of data, final approval.

\section{REFERENCES}

1. Ferlay J, Colombet $M$, Soerjomataram I, Mathers C, Parkin $D M$, Piñeros $M$, et al. Estimating the global cancer incidence and mortality in 2018: Globocan sources and methods. Int J Cancer 2019; 144(8):1941-53. doi: 10.1002/ijc.31937.

2. Tahir MZ. Low prevalence of colorectal cancer in South Asians than white population in UK: Probable factors. $J$ Coll Physicians Surg Pak 2018; 28(8):631-5. doi: 10.29271/jcpsp.2018.08.631.

3. Pan R, Zhu M, Yu C, Lv J, Guo Y, Bian Z, et al. Cancer incidence and mortality: A cohort study in China, 2008-2013. Int J Cancer 2017; 141(7):1315-23. doi: 10.1002/ijc.30825.

4. Murono K, Kawai K, Kazama S, Ishihara S, Yamaguchi H, Sunami E, et al. Anatomy of the inferior mesenteric artery evaluated using 3-dimensional CT angiography. Dis Colon Rectum 2015; 58(2):214-9. doi: 10.1097/DCR.0000000 000000285.

5. Zhao YE, Wang ZJ, Zhou CS, Zhu FP, Zhang LJ, Lu GM. Multidetector computed tomography of superior mesenteric artery: Anatomy and pathologies. Can Assoc Radiol J 2014; 65(3):267-74. doi: 10.1016/j.carj. 2013.10.001.

6. Gamo $E$, Jiménez $C$, Pallares $E$, Simón $C$, Valderrama $F$, Sañudo JR, et al. The superior mesenteric artery and the variations of the colic patterns. A new anatomical and radiological classification of the colic arteries. Surg Radiol Anat 2016; 38(5):519-27. doi: 10.1007/s00276-0151608-3.

7. Wang KX, Cheng ZQ, Liu Z, Wang XY, Bi DS. Vascular 
anatomy of inferior mesenteric artery in laparoscopic radical resection with the preservation of left colic artery for rectal cancer. World J Gastroenterol 2018; 24(32):3671-6. doi: 10.3748/wjg.v24.i32.3671.

8. Uh MK, Kandel J, Kitajewski J. Evaluating tumor angiogenesis. Methods Mol Biol 2013; 980:341-51. doi: 10.1007/978-1-62703-287-2_20.

9. Ivan CV, Mullineux JH, Verma R, Shah V, De A, Elabassy $M$, et al. Assessment of the inferior mesenteric vein diameter as a surrogate marker to evaluate response to neoadjuvant chemoradiotherapy for locally advanced rectal adenocarcinoma. Colorectal Dis 2017; 19(12): 1076-80. doi: 10.1111/codi.13811

10. Khan AN, Botchu R, Patel R, Elabassy M. Dilated SMV in colon cancer: Is there any significance. J Gastrointest Cancer 2012; 43(2):288-95. doi: 10.1007/s12029-0119302-0.

11. Edge SB, Compton CC. The American joint committee on cancer: The 7th edition of the AJCC cancer staging manual and the future of TNM. Ann Surg Oncol 2010; 17(6):1471-4. doi: 10.1245/s10434-010-0985-4.

12. Balyasnikova S, Brown G. Optimal imaging strategies for rectal cancer staging and ongoing management. Curr Treat Options Oncol 2016; 17(6):32. doi: 10.1007/ s11864-016-0403-7.

13. Mclnnes MD, Nanji S, Mackillop WJ, Flemming JA, Wei X, Macdonald DB, et al. Utilisation of pre-operative imaging for colon cancer: A population-based study. J Surg Oncol 2017; 115(2):202-7. doi: 10.1002/jso.24492.

14. Hashiguchi Y, Muro K, Saito Y, Ito Y, Ajioka Y, Hamaguchi $T$, et al. Japanese society for cancer of the colon and rectum (JSCCR) guidelines 2019 for the treatment of colorectal cancer. Int J Clin Oncol 2020; 25(1):1-42. doi: 10.1007/s10147-019-01485-z.

15. Van Cutsem E, Cervantes A, Adam R, Sobrero A, Van Krieken JH, Aderka D, et al. ESMO consensus guidelines for the management of patients with metastatic colorectal cancer. Ann Oncol 2016; 27(8):1386-422. doi: 10.1093/annonc/mdw235.

16. Nasseri $Y$, Langenfeld SJ. Imaging for colorectal cancer. Surg Clin North Am 2017; 97(3):503-13. doi: 10.1016/ j.suc.2017.01.002.

17. Hunter C, Brown G. Pre-operative staging of rectal cancer: A review of imaging techniques. Expert Rev Gastroenterol Hepatol 2016; 10(9):1011-25. doi: 10.1080/17474124.2016.1179577

18. Ma X, Shen F, Jia Y, Xia Y, Li Q, Lu J. MRI-based radiomics of rectal cancer: Preoperative assessment of the pathological features. BMC Med Imaging 2019; 19(1):86. doi: 10.1186/s12880-019-0392-7.

19. Uberoi AS, Bhutani MS. Has the role of EUS in rectal cancer staging changed in the last decade? Endosc Ultrasound 2018; 7(6):366-70. doi: 10.4103/eus. eus_36_18.

20. Sinkeet S, Mwachaka P, Muthoka J, Saidi H. ISRN Anat 2013; 2013:962904.

21. Momin AA, Chaubal NG, Saifi SG, Kazi ZN. Sonographic diagnosis of inferior mesenteric artery aneurysm and marginal artery of Drummond. J Clin Ultrasound 2008; 36:42-4.

22. Bruzzi M, M'harzi L, El Batti S, Ghazaleh RA, Taieb J, Poghosyan $T$, et al. Inter-mesenteric connections between the superior and inferior mesenteric arteries for left colonic vascularization: Implications for colorectal surgery. Surg Radiol Anat 2019; 41(3):255-64. doi: 10.1007/s00276-018-2139-5.

23. van Tonder JJ, Boon JM, Becker JH, van Schoor AN. Anatomical considerations on Sudeck's critical point and its relevance to colorectal surgery. Clin Anat 2007; 20(4):424-7. doi: 10.1002/ca.20417.

24. Yang KM, Lim SB, Yu CS, Kim JC. Severe distal ischemic proctitis with venous congestion following anterior resection for sigmoid colon cancer: The importance of Sudeck's point. Int J Colorectal Dis 2016; 31(5):1051-2 doi: 10.1007/s00384-015-2381-90. 